\title{
Are Wistar Rats the Most Suitable Normotensive Controls for Spontaneously Hypertensive Rats to Assess Blood Pressure and Cardiac Structure and Function?
}

\author{
Nazareth Novaes Rocha1 ${ }^{\text {(D) }}$ \\ Universidade Federal Fluminense (UFF), ${ }^{2}$ Niterói, RJ - Brazil \\ Editorial referring to the article: Is the Wistar Rat a more Suitable Normotensive Control for SHR to Test Blood Pressure and Cardiac Structure \\ and Function?
}

The first animal models of hypertension involved constriction of renal arteries (the Goldblatt kidney) or parenchyma (the Page kidney). Although these human analogs closely mimicked the pathophysiology of the disease, renovascular hypertension and the Page kidney represent only a small fraction of human hypertension.

Most experimental studies of hypertension using animals have focused on understanding the mechanisms of primary hypertension. Models of primary hypertension have been more difficult to develop. Some models may have greater face validity than others with respect to phenotypic aspects of hypertension such as age at onset, temporal course, severity, variability, and associated comorbidities. Given the clinical importance of hypertension-related target organ damage, it is noteworthy that models exhibiting face validity for left ventricular hypertrophy, metabolic abnormalities, heart failure, renal damage, and stroke (e.g., spontaneously hypertensive rats [SHR] and Dahl salt-sensitive [DSS] rats) are also available. However, other conditions such as spontaneous development of atherosclerosis or acute myocardial infarction are not typically observed in current models.

Several rat genetic models of hypertension have been used in genetic, (patho)physiological, and pharmacological studies. Rat strains exhibiting genetic hypertension include the SHR, DSS rat, the fawn-hooded hypertensive (FHH) rat, the Milan hypertensive strain,

\section{Keywords}

Animal, Rats, Wistar; Epigenesis,genetic; Blood Pressure; Hypertension/genetic; Constriction/pathologic; Renal Artery; Heart Function Tests. the Lyon hypertensive rat, the Sabra hypertensive rat, the genetically hypertensive rat, and the inherited stressinduced arterial hypertension rat model. Of these, the most studied is the SHR. In the past 10 years, more than 4,500 articles have been indexed in PubMed under the term spontaneously hypertensive rats. In contrast, the next most-cited model, the DSS rat, has been indexed 585 times over the same time span, and the other genetic rat strains indexed less frequently. ${ }^{1}$

Most of the genetically hypertensive rats have been derived from outbred Wistar or Sprague-Dawley (SD) breeding stocks with selection of hypertension-related traits. The strain of SHR originated in Kyoto, Japan, from the cross of an outbred Wistar male rat, which exhibited spontaneously elevated blood pressure, and a female rat with slightly elevated blood pressure. Subsequent brother-sister mating was continued with selection of animals with systolic blood pressure $>150 \mathrm{~mm} \mathrm{Hg}$. SHR are the pure line isolated from Wistar rats, which spontaneously showed stable hypertension symptoms in inbreeding offspring and developed high blood pressure at $7-15$ weeks of age. ${ }^{2}$

As controls for the SHR, most workers have used normotensive descendants of Wistar rats of the colony from which the SHR strain was derived (WistarKyoto rats, WKY). But the presumption that WKY are serviceable controls for SHR rests on the tacit assumption that all WKY constitute a single inbred strain. It appears, however, that whereas the National Institutes of Health distributed breeding stocks of SHR after they had been fully inbred (i.e., after 20 generations of brothersister mating), the breeding stocks of WKY had been distributed before they were fully inbred. Accordingly, the biological variability of WKY may be greater than that of SHR. Kurtz and Morris demonstrated that WKY

Mailing Address: Nazareth Novaes Rocha

Universidade Federal Fluminense. Rua Ernani Mello, 101. Postal Code: 24210-130, RJ - Brazil.

E-mail: nn_rocha@hotmail.com 
rats, a common genetic control for the SHR, had profound differences in phenotypes (growth rate and blood pressure) when obtained from different commercial suppliers. Moreover, all control strains are necessarily limited in the absence of a complete understanding of the genetic differences between the control and hypertensive strains. This emphasizes the importance of careful identification and consistent use of strains when performing experiments. Equally important are environmental influences on phenotypes. ${ }^{3}$

Rezende et al., ${ }^{4}$ evaluated the use of Wistar rats (WIS) and Wistar WKY as controls for SHR by assessing the long-term behavior of blood pressure and cardiac structure and function in these strains. Their main findings were: 1) blood pressure values in WKY were intermediate between SHR and WIS and close to hypertension borderline. WIS showed pressure values that were more consistent with those expected for normotensive rats; and 2) WKY presented earlier reductions in cardiac function when compared to WIS. Nevertheless, this work had some limitations; the authors did not perform an echocardiographic examination in the eighth week, i.e., the pre-hypertensive stage. Furthermore, the animals' food intake was not monitored, which may have affected weight gain, with the WIS group showing higher body mass. ${ }^{4}$

During the last years there has been enormous growth in the availability and quality of tools for genetic analysis. While such tools emerged more rapidly for humans, they now exist in a well-developed state for rats. However, although it has been recognized that the ideal control would have been a strain of rats identical to the SHR except for the genes for increased blood pressure, this is still away from reality.

The choice of a suitable animal model with spontaneous versus induced hypertension will depend on a number of factors, including the specific experimental question and the investigator's expertise available resources. No individual model will recapitulate all features of human hypertension and, regarding control models, a consistent use of strain from a reliable and authorized supplier should be considered. All these factors must be taken into account in the design and interpretation of experiments. ${ }^{5}$

\section{References}

1. Lerman LO, Kurtz TW, Touyz RM, Ellison DH, Chade AR, Crowley SD et al. Animal Models of Hypertension: A Scientific Statement From the American Heart Association. Hypertension. 2019 Jun.;73(6):e87-e120. doi: 10.1161/HYP.0000000000000090.

2. Zhang-James Y, Middleton FA, Faraone SV. Genetic architecture of Wistar-Kyoto rat and spontaneously hypertensive rat substrains from different sources. Physiol Genomics. 2013;45(13):528-38. doi: 10.1152/ physiolgenomics.00002.2013.

3. Kurtz TW, Morris RC Jr. Biological variability in Wistar-Kyoto rats: implications for research with the spontaneously hypertensive rat. Hypertension. 1987;10(1):127-31. doi: 10.1161/01.hyp.10.1.127.

4. Rezende LMT, Soares LL, Drummond FR, Suarez PZ, Leite L, Rodrigues JA et al. Is the Wistar Rat a more Suitable Normotensive Control for SHR to Test Blood Pressure and Cardiac Structure and Function? Int J Cardiovasc Sci. 2022; 35(2):161-171. Doi: https://doi. org/10.36660/ijcs.20200367.

5. Padmanabhan S, Joe B. Towards Precision Medicine for Hypertension: A Review of Genomic, Epigenomic, and Microbiomic Effects on Blood Pressure in Experimental Rat Models and Humans. Physiol Rev. 2017 Oct.;1;97(4):1469-528. doi: 10.1152/physrev.00035.2016. 